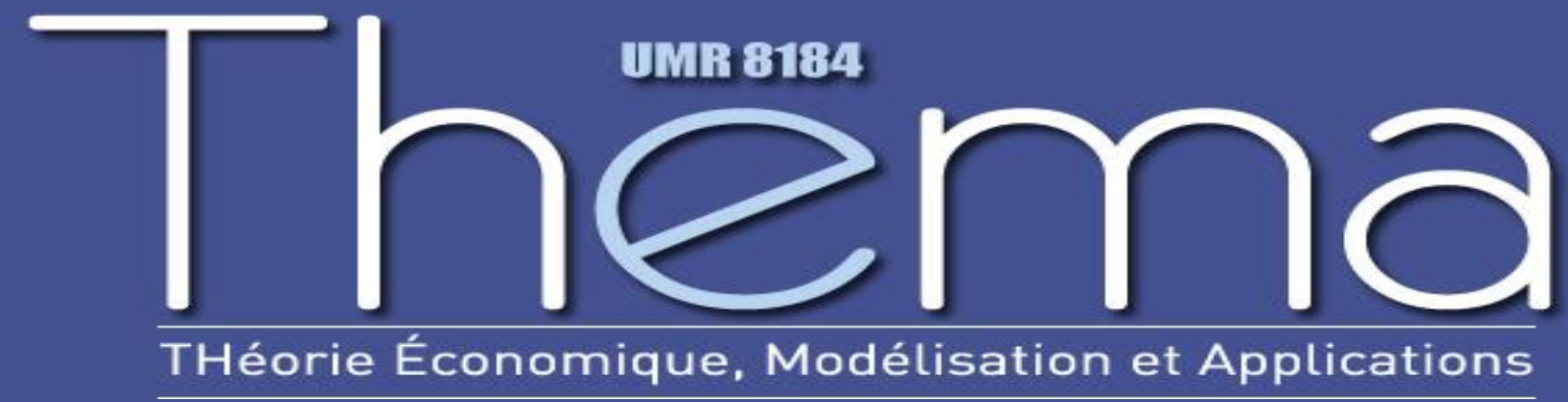

Thema Working Paper $n^{\circ}$ 2011-13

Université de Cergy Pontoise, France

A comparison between the methods of apportionment using power indices: the case of the U.S. presidential elections

Fabrice Barthelemy Mathieu Martin

June, 2011 


\title{
A comparison between the methods of apportionment using power indices: the case of the U.S. presidential elections
}

\author{
Fabrice BARTHÉLÉMY and Mathieu MARTIN* \\ THEMA \\ University of Cergy Pontoise \\ 33 boulevard du Port \\ 95011 Cergy Pontoise Cedex
}

November 30, 2010

Summary: In this paper, we compare five well-known methods of apportionment, the ones by Adams, Dean, Hill, Webster and Jefferson. The criteria used for this comparison is the minimization of a distance between a power vector and a population vector. The power is measured with the well-known Banzhaf power index and the populations are the ones of the different States of the U.S. We first explain under which conditions this comparison makes sense. We then compare the apportionment methods in terms of their ability to bring closer the power of the States to their relative population. The U.S. presidential election by Electors is studied through 22 censuses since 1790. Our analysis is largely based on the book written by Balinski and Young (2001). The empirical findings are linked with theoretical results.

JEL classification: C7, D7

Keywords: Banzhaf index, methods of apportionment, distances, balance populationpower.

\footnotetext{
${ }^{*}$ The authors thank Vincent Merlin and Ashley Piggins for all their comments.
} 


\section{Introduction}

Since 1790, the President of the United States of America is elected by an electoral college. The direct election by citizens was excluded (to avoid tumult and disorder) as the election by the Congress (to avoid the President being too dependent on this powerful institution).

There are two steps in the U.S. presidential process. Firstly, the citizens of every state choose the Electors. If a candidate obtains a majority in a State, he takes all of the electoral votes of this State (this "winner-take-all" method is generally true except for two States, Maine and Nebraska ${ }^{1}$ ). Secondly, the Electors vote for the President. The winner of the U.S. presidential election is the candidate who receives a majority of votes from the Electors. In 2000, there were 538 Electors divided into 50 States plus the District of Columbia. The number of Electors for each State is the sum of two components: a fixed one and a proportional one. The fixed one is the number of U.S. Senators which is always 2 for every State. The proportional component corresponds to the number of seats in the House of Representatives allocated to each State. For instance, in 2000, the State of California had 54 seats in the House of Representatives and 2 Senators, which leads to 56 Electors for the presidential election.

The paper focuses on the proportional component. Even if the number of representatives depends on the population of the State, the Constitution does not specify any exact rule to apportion the number of Electors to the different States. The crucial problem comes from the choice of this rule, because different apportionments can be obtained by using different methods. For example, consider the 1980 census, where Colorado population represents $1.279 \%$ of the whole U.S. population. With a total number of seats equals to 435 , an egalitarian apportionment, which should be as close as possible to the ideal one man-one vote, implies that Colorado gets 5.564 seats. Unfortunately, we can not divide a seat. Hence, should Colorado receive 5 or 6 seats (or, eventually some other number)? As the same question arises for each State, the apportionment issue becomes complex.

Moreover, many apportionment methods have been developed in the literature. Obviously, the choice of the method may have fundamental consequences. For instance, with the 1920 census, the State of New York can obtain 41 seats with one method and 45 with another. This difference is significant because it may lead to the election of another President.

\footnotetext{
${ }^{1}$ Maine and Nebraska both use the congressional district method. These two States give an Elector to the winner in each congressional district and two Electors to the statewide vote.
} 
As an illustration, President Hayes obtained 185 votes in 1876, and his opponent, Tilden, obtained 184 votes. This example underlines clearly the importance of the apportionment issue.

Every 10 years, since 1790, there is a census in U.S., which gives the number of inhabitants in every State. Since 1790, the proportional repartition of seats is made according to the most recent census and according to an apportionment method (chosen in an ad hoc way). Each State obtains at least one seat (according to the Constitution), which leads to at least three Electors in every State.

The names of the methods of apportionment are generally associated to famous American politicians (John Quincy Adams, Thomas Jefferson, Alexander Hamilton, Daniel Webster), which underlines the importance of the problem. The statesmen have proposed different methods to reflect the evolution of the society as the number of States and also the total population increased dramatically over the period 1790-2000. The size of the House of Representatives was 105 seats in 1790 , while there are 435 seats in 2000 . In the same way, the number of States has increased from 15 in 1790 to 50 in 2000. Finally, the number of Electors which was 538 in $2000^{2}$, was only 135 in 1790.

If the politicians have used apportionment in an empirical sense, technical developments have been made by scientists (several of them have proposed their own method, for instance James Dean or Joseph A. Hill). The latter have conducted the normative analysis of those methods. Clearly, the perfect method does not exist, a fact which is known since Webster at the beginning of the nineteenth century. However, Balinski and Young (2001) argue that the method of Webster, from a normative point of view, is better than the others. This method belongs to the well-known category of the divisor methods. Obviously, other methods can be found in the literature, but they are weak from a normative point of view.

The divisor methods are based on a particular number, called the ideal divisor. Keeping in mind the ideal one man-one vote, each inhabitant of the U.S. should have the same part of a seat whatever State he belongs to. In another word, whatever the State, a seat should be related to the same number of inhabitants. This number of inhabitants per seat corresponds to the ideal divisor. Hence, the sum of each State population divided by this divisor, and rounded according to the chosen method, must be equal to the predetermined number of seats (the House of Representatives size). When the number of Representatives is given (for

\footnotetext{
${ }^{2}$ The 435 seats plus the 100 senators and the 3 Electors for the District of Columbia (since 1961).
} 
example 435 in 2000), a brief calculus with an algorithm gives the divisor. The method of Webster rounds the quotient population/divisor to the nearest integer number. In the same spirit, the method of Jefferson rounds the quotient population/divisor to the integer part of this number, whereas the method of Adams rounds the quotient population/divisor to the smallest integer containing this quotient. Other methods are proposed in the literature, in particular the methods of Dean and Hill, detailed below.

Balinski and Young (2001) proposed several arguments which imply that Webster is better than the others from a normative point of view. Firstly, an Alabama paradox cannot appear when using this method. An Alabama paradox occurs when a State gets less seats when the total number of seats increases. Secondly, the apportionment with the Webster method is such that the number of seats for each State is near the quota (the quota of the State is equal to the population of this State divided by the whole population, multiplied by the total number of seats). Thirdly, we can show that the Webster method does not systematically advantage the smallest States or the largest ones: in the apportionment theory vocabulary we can say that there is no bias. Despite clear normative qualities, the method of Webster was only used to constitute the House of the Representatives in 1840, and from 1910 to 1930. In this paper, only the five most famous divisor methods are analyzed: the methods of Adams, Dean, Hill, Webster and Jefferson. The other well known method of Hamilton, or method of largest remainders, is not studied because it admits the possibility of an Alabama paradox. This constitutes a sufficient normative failure to reject it outright, even if it was used in the U.S presidential election.

The main question of this paper is the following: which method of apportionment offers the best balance between a State's population and its voting power? This question seems to be of crucial importance, since to get $10 \%$ of the Representatives does not mean that you get $10 \%$ of the power. Following Felsenthal and Machover (2001) or Leech (2002), we consider the power of a State and not its relative weight.

An essential problem is then to define power and to measure it. An interesting tool is given by the theory of power indices. This field has emerged at the intersection between cooperative game theory and political sciences. The literature on power indices is abundant and we can not summarize it in this paper. Elegant presentations are given in Felsenthal and Machover (1998), Laruelle (1998) or Laruelle and Valenciano (2008), among others.

For theoretical reasons (developed below), we only use the power index introduced by 
Banzhaf (1965). The power of a player is the probability that he should be a decisive player, that is to say a player such that when he belongs to a winning coalition (a group of players), the coalition wins, otherwise the coalition loses.

A good balance between population and power implies that each individual gets the same power. In other words, every individual should have the same probability of being decisive in their State and the State is decisive in the whole country. Obviously, all the individuals in a State have the same power but the States have different power since the number of Representatives may be different for two States. The choice of the power index is based on a probabilistic hypothesis that we impose on individual behavior, or on State behavior. In this way, we can try to reconciliate the theory of power index with the apportionment literature.

Unfortunately, there are technical difficulties when we compare population and power. Indeed, we have to use a notion of distance between two vectors. There exists an infinity of possible distances, the most famous being the euclidian distance which is particularly studied in this paper. Moreover, all the $L_{k}-$ norm, $\left.k \in\right] 0, \infty[$, are analyzed which is more general than the literature. Obviously, the results depend on the choice of $k$. One purpose of the paper is to determine which method of apportionment minimizes the distance between the population vector and the power vector.

An immediate question arises: why do not determine directly the apportionment which minimizes such a distance, without using a method of apportionment? This approach, for instance used by Leech (2002), has not been examined in the literature from a normative point of view. We show, in this paper, that such a method can produce an Alabama paradox. This weakness is certainly a sufficient condition to exclude this method as we do for the method of Hamilton.

In the second section, we first present the tools used in this paper: the methods of apportionment and the power indices. We explain then why we only use the Banzhaf power index for the comparison. In the third section, for each of the 22 U.S. censuses, we calculate which method of apportionment permits the best balance between population and power, in the classical majority case. The ranking of the methods is also computed. For instance, we show that the method which has a bias in favor of the largest States (Jefferson) is always ranked last. In section 4, we extend the results analyzed in sections 2 and 3 to the other cases than the majority. Clearly, the choice of the $\alpha$-majority may influence the 
ranking of the methods. The main idea is the following: the best method in a normative point of view is the one proposed by Webster. But sections 2 and 3 also underline that this method is not ranked first in general when the majority game is considered. Hence, may be there exists an $\alpha$ such that the method of Webster is ranked first. Since $\alpha$ is not fixed, studying all the distances would be too tedious. Hence, we focus on the standard euclidian distance. However, other distances are tested briefly. In theses three sections, we focus our analysis on the House of Representatives, as it corresponds to the proportional part of the apportionment (the other part corresponds to the fixed number of 2 senators, whatever the State). Then, in section 5, we take into account all the Electors and not only the ones obtained with the proportional part. This implies a modification of all our results since an important weight is given, proportionally, to the smallest States. In section 6 , the direct distribution of seats by just minimizing a distance between two vectors (population and power) is analyzed. We show that this approach admits the possibility of an Alabama paradox, as underlined previously. Section 7 concludes.

\section{Tools and methodology}

The purpose of the theory of apportionment is to distribute a fixed number of seats to several States proportionally to their population. From a mathematical point of view, the main problem in this theory comes from manipulation of integer numbers, since a seat cannot be divided. The objective is clearly different in the power indices theory. The goal is to measure the probability of an individual (or a State) being a decisive player in a coalition. Indeed, this is the metric used to measure the a priori influence of agents with power indices. Our approach consists in minimizing, for a State, the difference between its population and its power. For instance, if a State represents $25 \%$ of the whole population, the apportionment should be such that it owns about $25 \%$ of power. The direct comparison between population and power is not common in the literature: exceptions are Leech (2002), Bisson, Bonnet and Lepelley (2004) or Barthélémy and Martin (2007). 


\subsection{Apportionment}

\subsubsection{Preliminaries}

An apportionment method is given by a vector of population $\mathbf{p}=\left(p_{1}, \ldots, p_{n}\right)$ of $n$ States and a total number of seats $a>0$, which has to be distributed among these $n$ States. A vector $\mathbf{a}=\left(a_{1}, \ldots, a_{n}\right)$ is an apportionment of $a$, with $a_{i}$ a positive integer and $\sum_{i=1}^{n} a_{i}=a$. For example, in 2000, there were $a=435$ seats in the House of Representatives to be distributed among $n=50$ States. Moreover, constraints can be imposed on the apportionment. For instance, every U.S. State has at least one seat.

The quota for State $i$ is its share in the total population, multiplied by the total number of seats. Let $q_{i}$ be the quota, with $q_{i}=\frac{p_{i}}{\sum_{j=1}^{n} p_{j}} a$.

For example, if a State has a population equal to 100000 citizens in a country with 1000000 citizens, a number of seats equal to 100 implies a quota of 10, for this State. However, in general, the quota is not an integer (suppose that the number of seats is equal to 101 in our example), while the number of seats for a State has to be an integer. This difficulty lies at the heart of the theory of apportionment.

\subsubsection{The main methods of apportionment}

Apportionment is of crucial importance since it plays an important role in modern democracies, a classical example being the U.S. presidential election. There have been many debates since the 1787's U.S. Constitution and several methods have been proposed, not by scientists, but by famous American politicians. We do not develop here an historical presentation of the theory of apportionment, as this presentation is already made in the Balinski and Young's monograph (2001). Only the methods used in U.S. presidential elections are described in this section. 


\section{The method of Hamilton}

The apportionment is made easily: compute the quota and give to each State its integer part. Give any seat unapportioned to the States with the largest remainder. This method is not really considered in this paper because of its normative weakness (see Balinski and Young (2001)). However, it was used in the U.S. from 1850 to 1900, and so is important from a historical perspective. The method proposed by Hamilton in 1792 seems to be natural and simple, but it is not the first method proposed in U.S. Instead of considering the quota approach, as in the remainders methods, the number of citizens associated to one seat was first considered which corresponds to the divisor methods.

\section{The divisor methods}

The five most famous divisor methods are studied here: the methods of Jefferson, Adams, Webster, Hill and Dean.

The vector $\mathbf{a}$ is a Jefferson apportionment, if and only if

$$
\forall i=1, \ldots, n, \quad a_{i}=\left\lfloor\frac{p_{i}}{x}\right\rfloor
$$

with $x$ a divisor such that $\sum_{i=1}^{n} a_{i}=a$ and $\lfloor y\rfloor$ the integer part of $y$. In other words, once $a$ is fixed, we have to determine the divisor $x$ such that the sum of the integer parts of the rates population/power is equal to $a$. For instance, if $p_{i} / x=3.22$, then the States $i$ gets 3 seats. This method was used from 1790 to 1830 for the U.S. House of Representatives.

The vector $\mathbf{a}$ is an Adams apportionment, if and only if

$$
\forall i=1, \ldots, n, \quad a_{i}=\left\lceil\frac{p_{i}}{x}\right\rceil
$$

with $x$ a divisor such that $\sum_{i=1}^{n} a_{i}=a$ and $\lceil y\rceil$ the smallest integer greater than or equal to $y$. The construction of the method of Adams is identical to the one of Jefferson, the only difference being the way of rounding an integer. For example, if $p_{i} / x=3.22$, then the State $i$ gets 4 seats while it would have get 3 seats with the method of Jefferson.

The vector $\mathbf{a}$ is a Webster apportionment, if and only if

$$
\forall i=1, \ldots, n, \quad a_{i}=\left[\frac{p_{i}}{x}\right]
$$


with $x$ a divisor such that $\sum_{i=1}^{n} a_{i}=a$ and $[y]$ the nearest integer from $y$. For example, if $y=0.51$, then $[y]=1$ and if $y=3.22$, then $[y]=3$. In the particular case where $y$ is an integer plus 0.5 , there are two solutions. Thus if $y=8.5$, then $[y]=8$ or $[y]=9$. This method was used for the House of Representatives in 1840, 1910 and 1930³. Let us remark that between two successive integers, the value which changes the rounding is the arithmetic mean:

$$
\forall y \in[n, n+1], \begin{cases}{[y]=n,} & \text { if } y<(n+(n+1)) / 2 \\ {[y]=n+1,} & \text { if } y \geqslant(n+(n+1)) / 2\end{cases}
$$

The two last methods, proposed by Dean and Hill, are similar to the method of Webster. The difference comes from the way of computing the mean. Instead of using the arithmetic mean, we use the harmonic mean for the method of Dean (the harmonic mean for 2 and 3 is equal to 2.4), and the geometric mean for the method of Hill (the geometric mean for 2 and 3 is equal to 2.45). The method of Hill has been used for the House of Representatives since 1940.

Even if there are other methods proposed in the literature (with or without divisor), they are not considered in this paper because of their normative flows (see Balinski and Young, 2001). Remark that for the five divisor methods we have presented, the rounding depends on a particular value between two successive integers as illustrated in table 1 where $\tilde{y}$ corresponds to rounded value of $y^{4}$.

Table 1. Rounding with divisor methods

\begin{tabular}{|c|c|}
\hline & $\tilde{y}=n$ if \\
\hline Adams & $n-1<y \leqslant n$ \\
Dean & $n<y \leqslant 2 /\left(n^{-1}+(n+1)^{-1}\right)$ \\
Hill & $n<y \leqslant(n(n+1))^{0.5}$ \\
Webster & $n<y \leqslant(n+(n+1)) / 2$ \\
Jefferson & $n \leqslant y<n+1$ \\
\hline
\end{tabular}

\footnotetext{
${ }^{3}$ Note that in 1920, there are two new States, two new seats but no new apportionment, the one of 1910 is used. Note that the lack of a new apportionment violated the Constitution.

${ }^{4}$ Theoretically, when the value of $y$ corresponds to the arithmetic, geometric or harmonic mean, two rounded values are possible, $n$ and $n+1$. These cases are not mentioned here.
} 
As $n<2 /\left(n^{-1}+(n+1)^{-1}\right)<(n(n+1))^{0.5}<[n+(n+1)] / 2<n+1$, these methods can be ranked on a left-right axis according to their particular value which determines the rounding. The ranking is therefore Adams, Dean, Hill, Webster and Jefferson.

\section{Comparison of the methods}

Since the methods are different by construction, they do not satisfy the same properties. Hence, a normative approach is useful in order to compare them. Even if we do not enumerate all the possible properties in this presentation (see Balinski and Young, 2001, for more details), we present the main reasons that show that the method of Webster is said to be better than the others.

Firstly, there does not exist a divisor method such that a State receives less seats than the integer part of its quota and, at the same time, another State receives more seats than the integer greater than its quota. For example, suppose that the quota of State $i$ is 3.45 and the quota of State $j$ is 8.25. It is not possible that State $i$ receives 2 seats and State $j$ 10 seats. However, State $i$ can receive 2 seats and not automatically 3 or 4 seats. We say that this method does not satisfy the property called "staying within the quota". There is no divisor method which stays within the quota for every problem of apportionment. The probability that Webster violates this property is negligible (see Balinski and Young (2001), p. 129). Furthermore, the method of Webster is the only divisor method which respects the property of being "near the quota". This property says that if a State gives one seat to another State, it is not possible that the new number of seats of these two States brings them simultaneously nearer their quota (for a more detailed presentation, see Balinski (2001), p. 129).

The method of Hamilton satisfies the property "staying with the quota", by construction. Nevertheless, this method is not monotone when we consider an increase in the number of seats. It seems to be legitimate that a State does not lose a seat when the total number of seats increases (with constant population). The Alabama paradox shows that several methods violate this principle, in particular the method of Hamilton. The 1880's census, with 299 seats, gives 8 seats to Alabama and 7 if the number of seats is $300^{5}$. With a divisor method, this kind of problem can not occur.

Because of the Alabama paradox, we have to abandon Hamilton's method. However,

\footnotetext{
${ }^{5}$ An elegant geometric presentation of Alabama paradox, among other paradoxes, is given by Bradberry (1992).
} 
we still have to choose a method among the divisor methods. An argument for the method of Webster is that it is the only divisor method "near the quota". Another important property satisfied by the method of Webster concerns a possible bias. It is certainly a negative characteristic if a method has a persistent bias in favor either the small States or the large States. There are several ways of measuring this bias, an absolute one (does a State receive always more seats than its quota?) and a relative one (does a State receive always more seats for one citizen than another State?). The only divisor method without bias is the method of Webster. This is a theoretical and an empirical result: it is a fundamental property of the Webster method (see Balinski and Young 2001).

\subsection{Voting games and power indices theory}

\subsubsection{Voting games}

Generally, the notion of influence or power is studied with the help of a cooperative game theory concept, the voting game. A voting game is a pair $(N, W)$ where $N$ is the set of players (with $|N|=n$, where $|A|$ means the number of elements in the set $A$ ) and $W$ the set of winning coalitions, which is the set of groups of players which can enforce their decision.

Let $a$ be the total number of seats and $a_{i}$ be the number of seats of the State $i$. Thus we have $a=\sum_{i=1}^{n} a_{i}$. In this paper, we only consider integer values for $a$ and the $a_{i}$ 's. An $\alpha$-majority game is $\left[\alpha ; a_{1}, \ldots, a_{n}\right]$, where $\alpha$ is an integer greater than $a / 2$. A coalition $S$ of States is winning (written $S \in W$ ) if and only if $\sum_{i \in S} a_{i} \geqslant \alpha$.

The most famous voting game is the majority game, which perfectly corresponds to U.S. presidential election, where $\alpha=\frac{a}{2}+1$ if $a$ is even and $\alpha=\frac{a+1}{2}$ if $a$ is odd. Sometimes, it is easier to consider $\alpha$ in proportion (for example $50 \%$ in the majority case) and this is denoted $\bar{\alpha}$, with $\bar{\alpha}=\alpha / a$.

\subsubsection{Power indices theory}

We present here the two most famous power indices in the literature, because of their normative qualities and their historical importance. The construction of the two power indices we present here is quite different but there is, in the two cases, a particular player called the decisive or the swing player ${ }^{6}$ which has a crucial role. Our presentation is concise.

\footnotetext{
${ }^{6}$ This player is called a pivotal player in the Shapley-Shubik index case.
} 
A comprehensive description is given in Felsenthal and Machover (1998), in Laruelle (1998), or in Straffin (1994) among others.

The Shapley-Shubik power index (1954) takes into account the formation of the coalition $N$ which contains all the players. The order of appearance in this coalition is important. Assume that the player 1 joins the coalition $N$ first. If $a_{1} \geqslant \alpha$, then this player is pivotal. Otherwise, assume that player 2 joins the player 1 in the coalition $N$. If $a_{1}+a_{2} \geqslant \alpha$, then player 2 is pivotal, otherwise assume that the player 3 joins them in the coalition $N$, and so on. Since an empty set is not a winning coalition, while $N$ is always a winning coalition, there exists one pivotal player according to the chosen order of appearance in $N$. Obviously, there is no reason to only consider one order of appearance: all orders are supposed to have the same probability of occurring. The Shapley-Shubik power index of player $i$ is then the number of times it is pivotal divided by the number of possible orders of appearance in $N$, which is $n$ !. Formally the Shapley-Shubik of player $i$ is

$$
\phi_{i}=\frac{\text { number of orders with i pivotal }}{n !}
$$

and we obtain

$$
\phi_{i}=\sum_{S \subseteq N} \frac{(s-1) !(n-s) !}{n !}[v(S)-v(S \backslash\{i\})]
$$

with $s$ the number of players in $S$ and $v(S)=1$ if $S$ is a winning coalition and $v(S)=0$ otherwise. Remark that $[v(S)-v(S \backslash\{i\})]$ is different from 0 only if the player $i$ is pivotal in $S$.

Banzhaf (1965) proposes another power index where order in $N$ is not important. Its manipulation then becomes easier. Firstly, one has to determine all the non empty $2^{n}-1$ possible coalitions and the number of times player $i$ is swing. If this number is divided by $2^{n-1}$ (that is the number of coalitions containing the player $i$ ), we obtain the non-normalized Banzhaf power index (also called the Penrose-Banzhaf index). If it is divided by the total numbers of swing players, we obtain the normalized Banzhaf power index. Its formula for the player $i$ is

$$
\beta i=\frac{\text { number of times } i \text { is swing }}{\text { total number of swing players }}
$$

or

$$
\beta_{i}=\frac{\sum_{S \subseteq N}[v(S)-v(S \backslash\{i\})]}{\sum_{j \in N} \sum_{S \subseteq N}[v(S)-v(S \backslash\{j\})]}
$$




\subsubsection{Why do we use Shapley-Shubik and Banzhaf power indices?}

The power of a player (a State, a citizen), can be interpreted as the priori probability of his swinging. The Banzhaf index corresponds to a probabilistic hypothesis called independence: every player votes a priori for a candidate $\mathrm{A}$ or for a candidate $\mathrm{B}$, independently of the choices of the other players with a probability equal to $1 / 2$. This hypothesis is an idealized representation of electoral situations with floating voters, where the probability of tight results is high (the higher the number of players, the higher this probability, which is an application of the central-limit theorem).

From a probabilistic point of view, the Shapley-Shubik index corresponds to an hypothesis called homogeneity: instead of voting for A or B with a probability $1 / 2$, the players choose randomly a probability $p_{i}$ to vote for $\mathrm{A}$ in a uniform law in $[0,1]$. In other words, if $p_{i}=0.8$ is chosen, we are likely to obtain a clear result in favor of $\mathrm{A}$ or $\mathrm{B}$. This situation is really different from the repartition $50 \%$ in favor of $\mathrm{A}$ and $50 \%$ in favor of $\mathrm{B}$ mentioned previously with the independence hypothesis. However, on average, there is no favored candidate. For a discussion of the probabilistic models behind the power indices, see Straffin (1977) or Berg (1999).

For the American presidential election case, the power of a citizen must be distinguished from the power of a State. The citizens and the States belong to different voting games which imply different decisive players. A first voting game, the Electors election, is defined at the level of the States. For a given State, a citizen may be a decisive player, all the players being the citizens of this State. A second voting game corresponds to the vote for the president where the players are the different States. In this game, a State may be a swing player, as defined previously. The power of a citizen is thus the probability to be a decisive player in his State multiplied by the probability that the State he belongs to, is a swing player in the presidential election. The choice of the index, for the citizens and for the States is obviously important.

Looking for the best population-power balance is similar to level out the power of every citizen in his constituency. In other words, in a two-step game (election in the State followed by a national election), every citizen should have the same probability to be decisive.

Theoretical results about States behavior are presented in the table 2 ( $\beta^{\prime}$ corresponds to the Banzhaf non-normalized index while $\beta$ corresponds to the Banzhaf normalized index). 
Table 2. Theoretical results

\begin{tabular}{|c|c|c|}
\hline $\begin{array}{c}\text { Hypothesis on vote } \\
\text { of the citizens } \\
\text { (index used) }\end{array}$ & $\begin{array}{c}\text { Hypothesis on vote } \\
\text { of the States } \\
\text { (index used) }\end{array}$ & $\begin{array}{c}\text { Theoretical } \\
\text { recommendation }\end{array}$ \\
\hline $\begin{array}{c}\text { Independence }\left(\beta^{\prime}\right) \\
\text { Homogeneity }(\phi)\end{array}$ & $\begin{array}{c}\text { Independence }\left(\beta^{\prime}, \beta\right) \\
\text { Independence }\left(\beta^{\prime}, \beta\right)\end{array}$ & $\begin{array}{c}\text { Square root } \\
\text { Proportionality }\end{array}$ \\
\hline
\end{tabular}

When citizen behavior is represented by the independence hypothesis (described above), Penrose $(1946,1952)$ proposes an apportionment rule. He shows that the power of a citizen, measured by the non-normalized Banzhaf index, is proportional to the inverse of the square root of the population of his State. As each State has a 1/2 probability to vote independently for $\mathrm{A}$ or $\mathrm{B}$, its behavior corresponds to the independence hypothesis. In other words, in this situation, the State's power is measured by the non normalized Banzhaf index. Of course, in this election, its power depends on its weight $a_{i}$. If the number of players is large enough and if their weights are allocated randomly without the domination of one player (to avoid the existence of a too important weight), Penrose observes that, by applying the law of large numbers, the normalized Banzhaf power index of this player is approximately proportional to the number of seats he has. Consequently, there is a good chance that equal power among the citizens is achieved at least approximately with an apportionment of the seats proportional to the square root of the population. This result is known as the Penrose square root law. Recent results in this subject include papers by Felsenthal and Machover (2001), Slomczynski and Zyczkowski (2006), Feix, Lepelley, Merlin and Rouet (2007). This law is often mentioned in the literature but recent research shows that the independence hypothesis is not satisfied from an empirical point of view. In particular when it is confronted to the electoral results over a long time period (see Gelman, Katz and Tuerlinckx (2002), Gelman, Katz and Bafumi (2004) for U.S. elections - president, senators and governors since the 50's). In our opinion, this research permits to reject this law in our context.

If we admit that citizens vote according to the homogeneity hypothesis in each State, and that each State vote independently from the others, we have a ground to justify a proportional apportionment from a power point of view. More precisely, assume that, for each election, in each State $t$, a probability $p_{t}$ for a voter to vote for $A$ is drown from $[0,1]$ according to the homogeneity assumption. Assume furthermore that the different States chose this probability $p_{t}$ independently ${ }^{7}$. Then the apportionment can be made with a propor-

\footnotetext{
${ }^{7}$ This assumption thus differs from the classical Shapley-Shubik model, where the same $p_{t}$ should have
} 
tional method instead of using the Penrose law. In this case, the power of a citizen within a State is measured by the Shapley-Shubik index and it is inversely proportional to the State population (by definition, the sum of the Shapley-Shubik index adds up to one). Since the State behavior comes from the independence assumption, its probability of being swing is given by the non normalized Banzhaf index. All in all, by using the Penrose approximation, equal treatment in term of power should be attained with a proportional repartition of the seats. This model (independence of the votes for the States and homogeneity in the States) is the more adapted to support theoretically the application of a proportional method and thus an apportionment with a divisor method. At the end, we can compare the different apportionment method on the basis of the proportionality between the indices vector of Banzhaf power and population.

Our model may seem unrealistic but, i) the model where the non normalized Banzhaf index is used at each of the two-tier is not realistic at all (see Gelman, Katz and Tuerlinckx (2002), Gelman, Katz and Bafumi (2004)), ii) in the U.S. there are high variations between States in terms of election results, and our model capture this stylized fact.

\section{Apportionment in U.S. since $\mathbf{1 7 9 0}$}

In this section, we determine which divisor method of apportionment minimizes the distance population-power when considering the 22 U.S. censuses from 1790 to 2000 . The apportionments and the populations can be found in Balinski and Young (2001). To measure the differences between population and power, different distances can be used. Our goal is to minimize

$$
d_{k}(x, y)=\left(\sum_{i=1}^{n}\left|x_{i}-y_{i}\right|^{k}\right)^{\frac{1}{k}}, x \in \mathbb{R}^{n} \text { and } y \in \mathbb{R}^{n}
$$

where $x$ is the population vector and $y$ the power vector. Note that we consider the $L_{k}-$ norm, $\left.k \in\right] 0,+\infty[$. The well-known euclidian distance corresponds to $k=2$ and the difference in absolute values to $k=1$. Of course, others distances exist but are rarely used in the literature. Clearly our results depend on this choice, but the choice of $k$ may be made answering to the following question: do we want to impose an important weight to the largest differences or not? If $k$ tends to 0 , it means that we impose a very important weight been used among the States for the same election. 
to the possible equalities between population and power for a State, even if there exists some large differences between the others. If $k$ tends to infinity, an important weight is given to the largest differences. In this point of view, $k=2$ seems to be a good compromise and this value is often taken account for geometric reasons. Our results are summarized ${ }^{8}$ in table 3 .

Table 3. Values of $k$ for which a method permits the best balance between population and power

\begin{tabular}{|c||c|c|c|c|c|}
\hline Years & Adams & Dean & Hill & Webster & Jefferson \\
\hline 1790 & ] $0 ; 2.125]$ & ] $0 ; 2.125]$ & ] $0 ; 2.125]$ & ] $2.125 ; \infty[$ & \\
1800 & ] $0 ; \infty[$ & & & & \\
1810 & ] $0 ; \infty[$ & & & & \\
1820 & ] $0.25 ; \infty[$ & & ] $0 ; 0.25]$ & & \\
1830 & ] $1.5 ; \infty[$ & ] $0 ; 1.5]$ & & & \\
1840 & ] $1.25 ; \infty[$ & ] $0 ; 1.25]$ & ] $0 ; 1.25]$ & ] $0 ; 1.25]$ & \\
1850 & ] $0.875 ; \infty[$ & & ] $0 ; 0.875]$ & ] $0 ; 0.875]$ & \\
1860 & ] $0.625 ; \infty[$ & ] $0 ; 0.625]$ & & & \\
1870 & {$[0.75 ; \infty[$} & ] $0 ; 0.75]$ & & \\
1880 & ] $1 ; \infty[$ & ] $0 ; 1]$ & ] $0 ; 1]$ & ] $0 ; 1]$ & \\
1890 & ] $2.25 ; \infty[$ & ] $0 ; 2.25]$ & ] $0 ; 2.25]$ & ] $0 ; 2.25]$ & \\
1900 & ] $1.75 ; \infty[$ & & & ] $0 ; 1.75]$ & \\
1910 & ] $0 ; \infty[$ & & & \\
1920 & ] $1 ; \infty[$ & ] $0.75 ; 1]$ & ] $0.75 ; 1]$ & ] $0 ; 0.75]$ & \\
1930 & ] $1.125 ; \infty[$ & & ] $0 ; 1.125]$ & ] $0 ; 1.125]$ & \\
1940 & ] $1.25 ; \infty[$ & & & ] $0 ; 1.25]$ & \\
1950 & ] $1.5 ; \infty[$ & ] $0 ; 1.5]$ & ] $0 ; 1.5]$ & \\
1960 & ] $1.875 ; \infty[$ & & & ] $0 ; 1.875]$ & \\
1970 & ] $2.25 ; \infty[$ & ] $0 ; 2.25]$ & & \\
1980 & ] $1.75 ; \infty[$ & & & ] $0 ; 1.75]$ & \\
1990 & ] $1.5 ; \infty[$ & & & ] $0 ; 1.5]$ & \\
2000 & ] $1.25 ; \infty[$ & ] $0.5 ; 1.25]$ & ] $0 ; 0.5]$ & ] $0 ; 0.5]$ & \\
& & & & \\
\end{tabular}

\footnotetext{
${ }^{8}$ As there is an infinity of $L_{k}-$ norm distances, there can not be all analyzed. For the values of $k$ between 0 and 4 , we use the following sequence with a 0.125 step, $k=\{0.125,0.250, \ldots, 4\}$. Then we use integers from 4 to 10, and then higher values were tested to identify the robustness of the results when $k$ tends to infinity. The degree of precision could certainly be improved and then the bound proposed in table 3 could be more precise. This would not change the structure of the results.
} 
This table may be interpreted as follows: in 1790, for any value of $k$ between 0 and 2.125, the smallest distance is given by the methods of Adams, Dean and Hill (for these three methods, the apportionment is the same in 1790). We obtain a different result with $k$ greater than or equal to 2.125. Indeed, for $k>2.125$, the method of Webster gives the smallest distance. Let us notice that for this census, the Jefferson method is never optimal, whatever the value of $k$ (this is represented by an empty cell in the table). In the same spirit, in 1830, the distance is minimal for $k \leqslant 1.5$ with the method of Dean. For a largest value of $k$, Adams always minimizes the distance.

If we consider the most important distance in the literature, $k=2$, Adams is, in general, the method which minimizes the difference between population and power. This remark does not hold for the years 1890 and 1970.

In our exercise, the method of Adams seems to play an important role and not only for the quadratic distance $(k=2)$. We can give an intuition to this result. This method has a systematic bias in favor of the smallest States while the method of Jefferson has a bias in favor of the largest States. With the method of Adams, the rounding is upward. Hence, the smallest States receive relatively more seats with the rounding than the largest States. This is the opposite of the Jefferson's method. Furthermore, in a majority game, the Banzhaf index has a bias in favor of the largest States. This result is intuitive and even if there does not exist a proof, in our knowledge, several studies show it. The first intuition is given by Straffin (1994), in two examples and footnotes (pp 1133-1134). A confirmation is given, for example, by Felsenthal and Machover (2001) or by Feix, Lepelley, Merlin and Rouet (2007) for the European Union. Hence, the combination of the method of Adams with the Banzhaf index tends to vanish this bias. This explains the good results obtained with the method of Adams at least for a value of $k$ sufficiently large. For the method of Jefferson, its bias in favor of the largest States added to the bias of Banzhaf index, implies that it never gives the best population-power balance.

We present now the ranking of the methods in term of population-power balance which will give a complementary piece of information. Indeed, a method may never lead to the best adequacy, but may be ranked second or third. Not all the results will be reported here $^{9}$. The first important remark is that the method of Jefferson is almost always ranked

\footnotetext{
${ }^{9}$ Results can be found in the file http:/www.u-cergy.fr/barthelemy/balanceUSCensus.pdf.
} 
last, except in 1800 for $k \geqslant 3.25$ and in 1960 for $k \leqslant 0.375$. This is coherent in view of the intuition described above.

For the other methods of apportionment, there is no remarkable trend, even if the method of Webster is often ranked just behind the method of Adams. For the particular case $k=2$, the method of Webster is ranked 13 times first or second (among the 22 censuses).

Remark that the ranking is not monotone with $k$. For instance, in 1950, the method of Webster is ranked third for $k \leqslant 1.375$, fourth for $k \leqslant 2.5$ and second for $k \geqslant 2.625$. Other similar cases exist, for example for the method of Dean in 1820, 1920 and 2000, for the method of Hill in 1920 and again for the method of Webster in 1820 and 1870.

\section{The importance of $\alpha$}

Our purpose in this section is to study when the ranking of the methods in term of distance changes when we change $\alpha$. This exercise is of course fictitious, as the majority rule is the current rule for the U.S. But playing with the value of $\alpha$ will give a first glance at the robustness of our result for super majority rules.

In the previous section, we study a great number of distances with a given $\alpha$. Since $\alpha$ is not fixed now, we have to specify a value for $k$, otherwise the number of possibilities would become too large. With respect to the literature, the euclidian distance is considered $(k=2)$. Nevertheless, as this choice is arbitrary, some results with others values of $k$ are studied as well.

Generally as $\bar{\alpha}$ increases, the best method that minimizes the euclidian distance changes in the following order, Adams, Dean, Hill, Webster and Jefferson. This order is clearly the one given in table 1 for the computation of the rounding. This order is respected (excepted in 1790 and 1810), even if the five methods are not always ranked first (in 1820, the order is Adams, Hill, Webster and Jefferson, Dean never being first), or if there are ties (two different methods may give the same apportionment).

Only one graph is presented here ${ }^{10}$, the one corresponding to 1870 where the five apportionments are ranked first successively, which is not the case in general. Figure 2 is read as follows: the $x$ axis corresponds to $\alpha$ in proportion $(\bar{\alpha}=\alpha / a)$ and the $y$ axis corresponds to the ranking, from 1 to 5 .

\footnotetext{
${ }^{10}$ All the results can be consulted in the file cited above.
} 


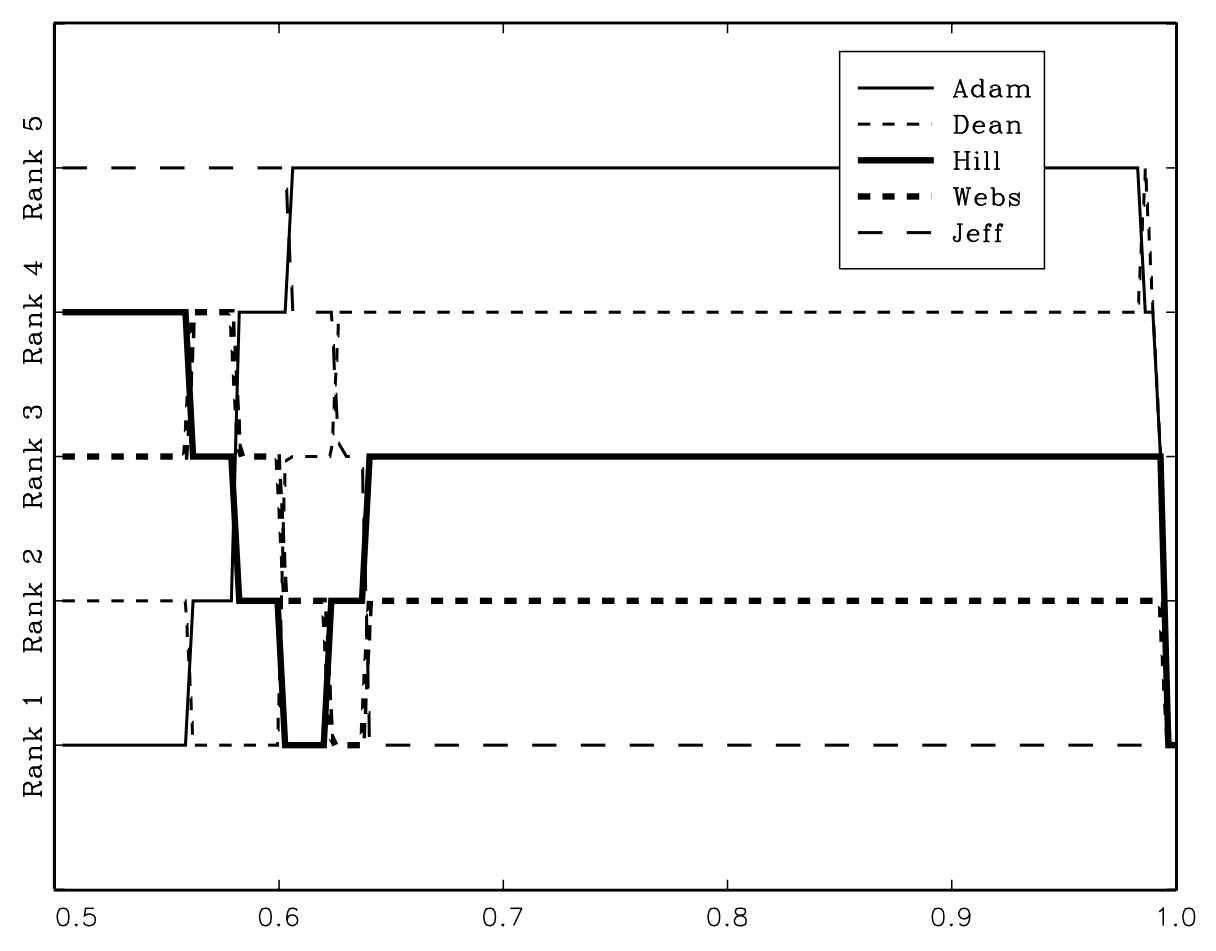

Figure 2. Ranking of the methods according to $\bar{\alpha}$ for year $1870(k=2)$

The different results for 22 censuses underlines the fact that there always exists an $\bar{\alpha}$ from which the method of Jefferson minimizes the euclidian distance. When $\bar{\alpha}$ is about $65 \%$ or more, the method which admits a systematic bias in favor of the largest States permits the best balance population-power. In the same spirit, in every census, an $\bar{\alpha}$ exists, around $60 \%$, such that the method of Webster minimizes the euclidian distance. Thus, there exists an $\bar{\alpha}$ such that the best method in a normative point of view minimizes the euclidian distance. In the previous section, we saw that empirical studies show a bias in favor of the largest States when we use the Banzhaf power index in a majority game. In the same way, the intuition suggests that there exists large values of $\bar{\alpha}$ such that the Banzhaf power index has a bias in favor of the smallest States. In between there exists a value of $\bar{\alpha}$ for which the Banzhaf index has no bias, and this value is often located between 60 and 65\%. Indeed, Feix, Lepelley, Merlin and Rouet (2007), or Slomczynski and Zyczkowski (2006), have shown that the proportionality between power and seats is better achieved with $\alpha=\sum_{i=1}^{N} a_{i}+\sqrt{1 / 4 \sum_{i=1}^{N} a_{i}^{2}}$. The corresponding $\bar{\alpha}$ value lies in the $[60 \%, 65 \%]$ interval in the European Union and the U.S. It is thus coherent that the best balance population-power should be obtained with the method of Webster for this $\alpha$. When $\bar{\alpha}$ increases, the Banzhaf 
index is in favor of the smallest States. As the method of Jefferson balances this bias, we logically find this method minimizes the distance between population and power.

Notice that there are some censuses for which the methods of Dean and Hill never minimize the euclidian distance. For example, this is the case in 1960, a period when the method of Hill has been used! Obviously, there always exists an $\bar{\alpha}$ for which all the methods minimize the distance simultaneously. Indeed, if $\bar{\alpha}$ tends to 1 , only one winning coalition arises (the grand coalition $N$ ), for which every State is swing. Then the power of the States does not depend on the apportionment.

Hence, when $k=2$, a general trend can be described even if particular situations may be observed.

We present now briefly other results when the distance is different from the traditional euclidian one. Two small values of $k$ are studied, $k=0.25$ and $k=1$ (the latter being as well quite used in the literature), and two large ones, $k=4$ and $k=8$.

When $k=0.25$, the instability of the results is evident. There are a lot of variations in the ranking and the curves are not as regular as seen for the euclidian distance case. However, there always exists an $\bar{\alpha}$ such that the method of Webster minimizes the distance. Moreover, for all the quotas greater than this particular value, the method of Jefferson minimizes the distance. When $k=1$, the results are the same with more regular curves.

The results for $k=4$ and $k=8$, are similar to the results for $k=2$. In this point of view, considering $k=2$ seems to be a relevant choice. The weight imposed to the large differences is sufficient (since the results are almost identical with higher values of $k$ ) and the curves are regular (there is a general simple trend, in the ranking).

\section{The Electors}

In this section, all the Electors are considered and not only the ones corresponding to the House of Representatives, which are determined by a method of proportionality. We have to add two seats to every State to the apportionments given by Balinski and Young (2001). Furthermore, we have to add the District of Columbia and its 3 seats. Indeed, since 1961, this new State has 3 Electors and this number does not depend on its population ${ }^{11}$.

\footnotetext{
${ }^{11}$ Note that Balinski and Young (2001) only consider the House of Representatives and thus do not give the population of the District of Columbia. We then consider the population given on the official census
} 
Adding these non proportional seats is not neutral. In this situation, the smallest States are overweight since their number of seats increases more in proportion. For instance, in 2000, adding 2 seats to Alaska corresponds to an increase of $200 \%$, while the same two seats corresponds to an increase of $3.8 \%$ for California. Thus, only the bias in favor of the largest States induced by the method of Jefferson can balance the excess weight of the smallest States. So, the method of Jefferson minimizes all the distances, except for scarce cases. For instance, when $k$ is slightly less than 1 , the methods of Adams or Webster minimize the distance. In other words, the bias in favor of the smallest States implied by the two non proportional seats is only balanced by the method of Jefferson, even if $\bar{\alpha}$ is small $(1 / 2)$. When adding only one seat to every State, the results remain nearly the same, although the predominance of the method of Jefferson is not so evident. In other words, adding some seats to the proportional apportionment change drastically the results. Obviously, this is particularly true in some situations where the populations are really different.

\section{Why do we use a method of apportionment?}

In this paper, we have shown which are the best classical methods of apportionment in order to minimize the difference between population and power. A natural question arises: why do we consider them when the optimal apportionment could be determined directly such that the distance population-power would be minimal? If we agree that this criteria of minimal distance is essential from a normative point of view, like the Alabama paradox or the bias, then an apportionment is possible without using a classical method as in the manner of Leech (2002).

We assume that the power vector should be equal to the population vector $\mathbf{p}$. We associate a power vector, denoted $\mathbf{b z}(\mathbf{a})=\left(b z_{1}(\mathbf{a}), \ldots, b z_{n}(\mathbf{a})\right)$ to each apportionment $\mathbf{a} \in$ $R^{n}$. We look for $\mathbf{a}^{*}$, which minimizes a distance between $\mathbf{p}$ and $\mathbf{b z}\left(\mathbf{a}^{*}\right)$. Like Leech (2002), we consider the euclidian distance $(k=2)$. Thus we have:

$$
\mathbf{a}^{*}=\operatorname{argmin}_{\mathbf{a}} \sum_{i=1}^{n}\left(b z(\mathbf{a})_{i}-p_{i}\right)^{2}
$$

Leech (2002), with an iterative procedure on real numbers shows that it is not necessary to know every possible apportionment. As the integer case is studied here, this iterative web site http://www.census.gov/prod/www/abs/decennial/. 
procedure can not be used, and all the apportionments have to be taken into account. Is this approach relevant when considering the normative properties mentioned before? We are going to answer to this fundamental question using the following example: let a voting game with $a=6$ and 3 players $^{12}$ in a majority game with populations in proportion equal to $p^{\prime}=\left(\begin{array}{lll}0.46 & 0.33 & 0.21\end{array}\right)$.

As shown in table 4 , the optimal apportionment in this case is $a_{1}=a_{2}=a_{3}=2$.

Table 4. Apportionment and distance population-power

\begin{tabular}{|c|c|c||c|}
\hline$a_{1}$ & $a_{1}$ & $a_{3}$ & Distance \\
\hline 6 & 0 & 0 & 0.4446 \\
5 & 1 & 0 & 0.4446 \\
4 & 2 & 0 & 0.4446 \\
4 & 1 & 1 & 0.4446 \\
3 & 3 & 0 & 0.0746 \\
3 & 2 & 1 & 0.0366 \\
$\mathbf{2}$ & $\mathbf{2}$ & $\mathbf{2}$ & $\mathbf{0 . 0 3 1 3}$ \\
\hline
\end{tabular}

When we consider 7 seats (details are omitted), two solutions are possible, $(3,2,2)$ and $(4,2,1)$, which lead to the same result. Below, the arrow means that the optimal solution $(2,2,2)$ obtained with six seats, becomes, with one more seat, $(3,2,2)$ or $(4,2,1)$. The States (the players) for which the number of seats changes are in bold:

- $(2,2,2) \longrightarrow(\mathbf{3}, 2,2)$

- $(2,2,2) \longrightarrow(\mathbf{4}, 2, \mathbf{1})$

Hence the second solution leads to an Alabama paradox.

When there are 8 seats instead of 7 , we obtain again 2 different solutions, $(4,2,2)$ and $(5,2,1)$ :

- $(3,2,2) \longrightarrow(4,2,2)$

- $(4,2,1) \longrightarrow(4,2,2)$

- $(4,2,1) \longrightarrow(5,2,1)$

When there are 9 seats instead of 8 , the State 1 loses at least 1 seat according to the solution used with 8 seats:

\footnotetext{
${ }^{12}$ We assume that $a_{1} \geqslant a_{2} \geqslant a_{3}$, the populations are ranked in decreasing order, $p_{1} \geqslant p_{2} \geqslant p_{3}$.
} 
- $(4,2,2) \longrightarrow(\mathbf{3}, \mathbf{3}, \mathbf{3})$

- $(5,2,1) \longrightarrow(\mathbf{3}, \mathbf{3}, \mathbf{3})$

Thus, with this method, an Alabama paradox can arise. Furthermore, the solution is not necessarily unique. For these reasons, as for the method of Hamilton, this method of apportionment should be rejected. This reinforces the approach we have proposed previously.

\section{Conclusion}

The number of Electors for each State of the U.S. is obtained principally via a proportional method of apportionment. Since the 1790 census, various methods were used for the apportionment. The purpose of the paper is to determine if one of them permits a better balance between population and power. From a normative perspective, this criterion seems important, as important as the bias or the Alabama paradox for instance. A good balance between population and power means that every citizen in the country has the same power whatever the State he belongs to, which seems to be a minimal condition of democracy.

Technical difficulties arise. Firstly, we have to measure the power. For that we use the tools of power indices theory. We only consider the Banzhaf index for technical and theoretical reasons. Secondly, since we want to balance population-power as much as possible, we have to compare two vectors with a notion of distance. We use the $L_{k}-$ norm distances with a particular attention to the standard euclidian distance, which is commonly used in the literature.

When considering only the proportional part of the Electors and a majority game, the method of Adams permits the best balance between population and power. If the different methods are ranked, the method of Jefferson is almost always ranked last. This result is intuitive since, for a majority game, Banzhaf has a bias for the largest States. The two bias are balanced to obtain the best proportionality. If we do not consider only majority games, the result changes drastically. Around a value of $62 \%$ (the majority being $50 \%$ ), the Banzhaf index has no bias and is then perfectly compatible with the method of Webster, the only method of apportionment without bias. This positive result means that there exists $\bar{\alpha}$ such that the best method from a normative point of view permits the best balance between population and power. If $\bar{\alpha} \geqslant 65 \%$, the method of Jefferson always minimizes the 
distances. If we consider all the Electors and not only its proportional part, the weight given to the smallest States is too high and it could only be balanced using the method of Jefferson.

In this paper, we compare the 5 most famous methods of apportionment from an empirical point of view. A theoretical study seems to be essential. But it is clear that it will be very difficult to obtain exact results, since the methods of apportionment and the Banzhaf index are technically awkward to manipulate. A possibility is certainly to obtain these results with simulations even if it should be difficult to obtain general trend. Indeed, the number of possibilities is huge when population, $\bar{\alpha}$ and the number of seats are allowed to vary.

\section{REFERENCES}

Balinski M., Young P. [2001], Fair Representation meeting the ideal of one man, one vote, Brookings Institution Press, Washington D.C, second edition.

BANZHAF J.F. [1965], "Weighted voting doesn't work A mathematical analysis", Rutgers Law Review, 19, p. $317-343$.

BARthélÉmy F., Martin M. [2007], "Critères pour une meilleure répartition des sièges du Val d'Oise au sein des structures intercommunales: une application au cas du Val d'Oise", Revue Économique, 58, p. $399-426$.

BerG S. [1999], "On voting power indices and a class of probability distributions: With Applications to EU Data", Group Decision and Negotiation , 8, p. $17-31$.

Bisson F., Bonnet J., Lepelley D. [2004], "La détermination du nombre de délégués au sein des structures intercommunales : une application de l'indice de pouvoir de Banzhaf', Revue d'Économie Rgionale et Urbaine, 2, p. 259 - 282.

Bradberry B.A. [1992], "A geometric view of some apportionment paradoxes", Mathematics Magazine, 65, p. $3-17$.

Feix M.R., Lepelley D., Merlin V., Rouet J.L. [2007], "On the voting power of an alliance and the subsequent power of its members", Social Choice and Welfare, 28, p. $181-207$.

Felsenthal D.S., Machover M. [1998], The measurement of voting power: theory and practice, problems and paradoxes, Edward Elgar Publishers, London. 
Felsenthal D.S., Machover M. [2001], "The treaty of Nice and qualified majority voting", Social Choice and Welfare, 18, p. $431-464$.

Gelman A., Katz J.N., Bafumi J. [2004], "Standard voting power indexes do not work: an empirical analysis", British Journal of Political Science, 34, p. 657 - 674.

Gelman A., Katz J.N., Tuerlinckx F. [2002], "The mathematics and statistics of voting power", Statistical Science, 17, p. $420-435$.

LARUELle A. [1998], Game theoretical analysis of decision-making processes with applications to the European Union, PHD thesis, Université Catholique de Louvain.

Laruelle A., Valenciano F. [2008], Voting and collective decision-making: bargaining and power, Cambridge University Press.

LEECH D. [2002], "Designing the voting system for the Council of the European Union", Public Choice, 113, p. $437-464$.

Penrose L.S. [1946], "The elementary statistics of majority voting", Journal of the Royal Statistical Society, 109, p. $53-57$.

Penrose L.S. [1952], On the objective study of crowd behaviour, London H.K. Lewis.

Shapley L.S., Shubik M. [1954], "A method for evaluating the distribution of power in a committee system", American Political Science Review, 48, p. 787 - 792.

Slomczynski W., Zyczkowski K. [2006], "Penrose voting system and optimal quota", Acta Physica Polonica B, 37, p. 3133 - 3143.

Straffin P.D. [1977], "Homogeneity, independence power indices", Public Choice, 30, p. $107-108$.

Straffin P.D. [1994], "Power and stability in politics", in Handbook of Game Theory with Economic Application, Volume 2 (Aumann. R.J and Hart. S, eds), Elsevier Science. 